\title{
Investigating the potential clinical benefit of Selumetinib in resensitising advanced iodine refractory differentiated thyroid cancer to radioiodine therapy (SEL-I- METRY): protocol for a multicentre UK single arm phase II trial
}

Sarah R. Brown ${ }^{1 *}$, Andrew Hall1, Hannah L. Buckley ${ }^{1}$, Louise Flanagan ${ }^{1}$, David Gonzalez de Castro², Kate Farnell ${ }^{3}$, Laura Moss ${ }^{4}$, Rebecca Gregory ${ }^{5}$, Kate Newbold ${ }^{6}$, Yong Du', Glenn Flux ${ }^{5}$ and Jonathan Wadsley ${ }^{8}$

\begin{abstract}
Background: Thyroid cancer is the most common endocrine malignancy. Some advanced disease is, or becomes, resistant to radioactive iodine therapy (refractory disease); this holds poor prognosis of 10\% 10-year overall survival. Whilst Sorafenib and Lenvatinib are now licenced for the treatment of progressive iodine refractory thyroid cancer, these treatments require continuing treatment and can be associated with significant toxicity. Evidence from a pilot study has demonstrated feasibility of Selumetinib to allow the reintroduction of I-131 therapy; this larger, multicentre study is required to demonstrate the broader clinical impact of this approach before progression to a confirmatory trial.

Methods: SEL-I-METRY is a UK, single-arm, multi-centre, two-stage phase II trial. Participants with locally advanced or metastatic differentiated thyroid cancer with at least one measureable lesion and iodine refractory disease will be recruited from eight NHS Hospitals and treated with four-weeks of oral Selumetinib and assessed for sufficient I-123 uptake (defined as any uptake in a lesion with no previous uptake or $30 \%$ or greater increase in uptake). Those with sufficient uptake will be treated with I-131 and followed for clinical outcomes. Radiation absorbed doses will be predicted from I-123 SPECT/CT and verified from scans following the therapy. Sixty patients will be recruited to assess the primary objective of whether the treatment schedule leads to increased progression-free survival compared to historical control data.

\footnotetext{
* Correspondence: S.Brown@leeds.ac.uk

${ }^{1}$ Leeds Institute of Clinical Trial Research, University of Leeds, Leeds LS2 9JT, UK

Full list of author information is available at the end of the article
}

(c) The Author(s). 2019 Open Access This article is distributed under the terms of the Creative Commons Attribution 4.0 International License (http://creativecommons.org/licenses/by/4.0/), which permits unrestricted use, distribution, and reproduction in any medium, provided you give appropriate credit to the original author(s) and the source, provide a link to the Creative Commons license, and indicate if changes were made. The Creative Commons Public Domain Dedication waiver (http://creativecommons.org/publicdomain/zero/1.0/) applies to the data made available in this article, unless otherwise stated. 
(Continued from previous page)

Discussion: The SEL-I-METRY trial will investigate the effect of Selumetinib followed by l-131 therapy on progression-free survival in radioiodine refractory patients with differentiated thyroid cancer showing increased radioiodine uptake following initial treatment with Selumetinib. In addition, information on toxicity and dosimetry will be collected. This study presents an unprecedented opportunity to investigate the role of lesional dosimetry in molecular radiotherapy, leading to greater personalisation of therapy. To date this has been a neglected area of research. The findings of this trial will be useful to healthcare professionals and patients alike to determine whether further study of this agent is warranted. It is hoped that the development of the infrastructure to deliver a multicentre trial involving molecular radiotherapy dosimetry will lead to further trials in this field.

Trial registration: SEL-I-METRY is registered under ISRCTN17468602, 02/12/2015.

Keywords: lodine refractory differentiated thyroid cancer, MEK inhibitor, Selumetinib, Radioiodoine therapy, Phase II, Dosimetry

\section{Background}

Thyroid cancer is the most common endocrine malignancy, but remains relatively rare despite incidence doubling since the 1990s. In 2014, 3400 people in the United Kingdom (UK) were diagnosed with thyroid cancer and in 2014 approximately 370 patients died from the disease [1].

\section{Current treatment}

A combination of surgery, radioactive iodine (RAI) therapy and long-term thyroid stimulating hormone (TSH) suppression cures many patients with differentiated thyroid cancer, namely papillary and follicular cancers. However around 10\% of patients develop advanced disease which will eventually become resistant to therapy. Prognosis for these patients is poor with a 10-year overall survival rate of $10 \%$ compared with $56 \%$ in patients with RAI avid metastases [2].

\section{Treatment for radioiodine refractory disease}

Attempts have been made in the past to restore RAI avidity with lithium carbonate [3] and retinoic acid [4], but results have been disappointing and have failed to show clear clinical benefit.

More recently a number of multi-targeted kinase inhibitors have been tested; two phase III trials have shown significant improvements in progression free survival (PFS) with Sorafenib and Lenvatinib when compared with placebo $[5,6]$. While these improvements are certainly to be welcomed, both agents cause toxicities, including fatigue, diarrhoea, hand-foot syndrome, weight loss and hypertension. Since they are taken continuously to disease progression this can have a significant impact on patients' quality of life.

\section{MEK and radioiodine refractory disease}

Mutations of RET, RAS or BRAF are present in approximately $70 \%$ of papillary thyroid cancers [7]. These oncogenes are part of the mitogen-activated protein kinase
(MAPKinase) signalling pathway. Preclinical data suggest that activation of this pathway is critical to tumour initiation and transformation, and also results in reduced expression of genes involved in iodide metabolism, in particular the sodium iodide symporter (NIS) which is responsible for the uptake of iodine by thyroid cells. For example, papillary thyroid cancers with BRAF mutations are associated with reduced expression of key genes involved in iodine metabolism, including NIS [8].

In vitro work with rat thyroid PCCL3 cells with induced BRAF mutations has shown that treating the cells with a MEK inhibitor restores expression of TSH receptors, NIS and thyroglobulin, indicating that the effects of BRAF mutation to reduce NIS expression are potentially reversible [9].

\section{Selumetinib}

Selumetinib is an oral, potent and highly selective, allosteric MEK1/2 inhibitor. MEK1/2 are critical components of the MAPKinase pathway. This pathway is frequently dysregulated due to activating mutations in various oncogenes, including RAS or RAF. Inhibition of MEK, which lies downstream of these targets, blocks inappropriate signal transduction offering a promising therapeutic strategy. Selumetinib is not currently approved for use in any clinical indications in humans, but phase I and II trials have been conducted in pancreatic cancer [10], melanoma [11-16], non-small cell lung cancer [17-19] and differentiated thyroid cancer [20-22] and it has recently been designated orphan drug status for treatment of differentiated thyroid cancer in the United States. The drug has been found to have an acceptable side effect profile; most frequently reported side effects include diarrhoea, nausea and vomiting, dyspnoea, blurred vision, tiredness and acneiform rash.

A previously reported pilot study [21] tested the use of Selumetinib $75 \mathrm{mg}$ twice daily for four weeks in patients with radioiodine refractory differentiated thyroid cancer. All participants who started therapy were able to complete 
the full prescribed course of Selumetinib without dose reductions or delays. No toxic effects of Grade 3 or above were noted. Of 20 participants evaluated, Selumetinib increased radioiodine uptake in 12 (60\%). Eight of these 12 achieved sufficient increased uptake for further radioiodine therapy to be viable (67\%). Of the eight participants treated with radioiodine, five (63\%) had partial responses and three (38\%) had stable disease. All participants had decreases in serum thyroglobulin level.

\section{Lesional dosimetry}

Standard practice in the UK, endorsed by recently published guidelines [23] is to prescribe an empirical activity, typically $3.7-7.4 \mathrm{GBq}$ iodine-131 (I-131) to patients with metastatic disease. It is well recognised that this leads to a wide range of absorbed dose delivered to tumour targets and organs at risk, and therefore very likely to lead to widely divergent outcomes. This is in stark contrast to modern external-beam radiotherapy practice where absorbed dose delivered to target volumes and to nearby organs at risk is prescribed and can be accurately measured. There have been calls to address this issue [24]. There is evidence that the absorbed dose delivered to thyroid remnants correlates with response for patients undergoing radioiodine ablation [25] and this study will investigate a similar relationship for patients with locally advanced or metastatic disease.

The previously described pilot study [21] used pretreatment iodine-124 (I-124) positron emission tomography (PET) to estimate absorbed dose to individual lesions. A minimum lesion absorbed dose of 20Gy was chosen as a cut off for proceeding with further I-131 therapy. The choice of cut off used in this pilot does not appear to have been made on the basis of any published data. Additionally the absorbed doses were not verified after treatment, unlike in this study.

\section{SEL-I-METRY trial}

The SEL-I-METRY trial has been designed to demonstrate the broader clinical impact of the use of Selumetinib therapy to re-sensitise radioiodine refractory thyroid cancer patients to treatment with radioiodine. The trial will address the dosimetry of I-131 therapy to investigate the relationship between the absorbed dose delivered and the clinical benefit from therapy. In addition the extent to which the therapeutic activity of I-131 can be individualised from the absorbed doses predicted in a pre-therapy tracer study will be analysed using dosimetric calculations. It will also explore possible biomarkers for response to this strategy, including measurement of post I-131 protein bound iodine (PBI) and molecular markers both in tumour tissue and circulating cell-free DNA.

\section{Methods \\ Design}

SEL-I-METRY is a UK, single arm, multi-centre, twostage phase II proof of concept trial investigating the use of Selumetinib to resensitise iodine refractory patients with differentiated thyroid cancer. To ensure at least 38 participants are treated with I-131, approximately 60 participants will be recruited from eight NHS Hospitals. Participants will receive $75 \mathrm{mg}$ of Selumetinib orally twice daily for four weeks (dose may be reduced due to adverse events) and will then be assessed for evidence of an increase in radioiodine uptake via iodine-123 (I-123) single photon emission computed tomography/computed tomography (SPECT/CT); those showing sufficient uptake will then receive I-131 therapy (further details on patient imaging and dosimetry can be found in Additional file 1). A previous pilot study [21] used I-124 sodium iodide (NaI) although there is currently no commercially available source of this isotope in the UK. This trial therefore elected to use I-123 as an alternative to determine changes in radioiodine uptake following Selumetinib therapy, and to predict likely lesional absorbed dose in participants proceeding to I-131 therapy. The use of I-123 as opposed to I-124 is not thought to pose any significant risk to the current trial; whilst theoretically there may be advantages to using I-124, there are no known clinical comparator studies between the two techniques and a pragmatic decision to use I-123 was deemed reasonable by the trial team. An early stopping rule is incorporated in the case where none of the first 10 patients treated with Selumetinib show sufficient iodine uptake to progress to I-131 therapy; should this happen the trial may be discontinued. The study protocol and this manuscript have been written in accordance with Standard Protocol Items: Recommendations for Interventional Trials (SPIRIT) guidelines [26] (checklist included as an Additional file 2).

\section{Ethical approval}

The trial has received ethics approval from the NHS National Research Ethics Service (NRES) East MidlandsLeicester South (15/EM/0455).

\section{Primary objective}

This trial aims to assess the efficacy of Selumetinib followed by I-131 therapy in iodine refractory patients with differentiated thyroid cancer demonstrating increased iodine uptake following initial treatment with Selumetinib. The primary objective is to assess whether the treatment schedule leads to increased PFS compared to historical control data. PFS has been chosen as the primary endpoint assessing efficacy to allow for proof of concept in this Phase II trial to be obtained in a quick and robust manner and as it is clinically relevant to patients. 


\section{Secondary objectives}

- Assess the safety and toxicity of Selumetinib;

- Assess efficacy based on response and overall survival of Selumetinib followed by I-131 therapy;

- Determine the rate of iodine uptake in metastatic lesions in iodine refractory patients with differentiated thyroid cancer treated with Selumetinib.

\section{Exploratory objectives}

- Investigate the role of lesional dosimetry using I-123 SPECT/CT to predict response to I-131 therapy;

- Assess correlation between PBI with absorbed doses delivered and treatment outcome in patients who receive I-131;

- Summarise BRAF, NRAS and TERT gene mutational status, and additional mutational analysis of relevant genes, from tissue and circulating cell-free DNA;

- Assess patient reported quality of life.

\section{Sample size}

Based on previous data, median progression free survival is estimated to be approximately six months, corresponding to $25 \%$ of patients alive and progression free at 12 months [5]; a clinically important increase suggesting further investigation of Selumetinib (assuming exponential survival) is deemed to be an increase to $44 \%$ (hazard ratio $=0.6$ ). Using a 1 -sided $10 \%$ significance level, and with $80 \%$ power, 38 patients with sufficient radioiodine uptake following Selumetinib therapy are required (allowing for $10 \%$ drop out), based on a Case and Morgan two-stage design [27]. Assuming sufficient increase in iodine uptake to warrant I-131 therapy in $60 \%$ of patients [21], approximately 60 patients will be recruited in total.

A formal interim analysis will be performed after 21 participants with sufficient iodine uptake following Selumetinib treatment have been recruited and after a minimum of 12 months recruitment. If there is evidence to suggest that 12 month progression free survival is no better than 25\% based upon the Nelson-Aalen estimator then the trial may be terminated early for lack of activity.

If none of the first 10 participants treated with Selumetinib has sufficient increase in iodine uptake to progress to I-131 therapy, then the trial may be discontinued. This corresponds to excluding an uptake rate of at least $26 \%$ with $95 \%$ confidence.

\section{Eligibility criteria}

Inclusion and exclusion criteria can be found in Table 1. Patients with Gilbert's syndrome (persistent or recurrent hyperbilirubinemia that is predominantly unconjugated in the absence of evidence of haemolysis or hepatic pathology) will be eligible. Eligibility waivers will not be granted in this trial.

\section{Consent, screening and registration}

Written informed consent will be obtained prior to the commencement of all trial assessments, tests and procedures by authorised members of the trial team. Screening investigations will be conducted to confirm eligibility following consent and prior to registration. These investigations will include: CT neck, chest, abdomen and pelvis (without iodinated contrast); echocardiogram/multiple gated acquisition (MUGA); single 12-lead electrocardiogram (ECG); vital signs; ophthalmology examinations (best corrected visual acuity; intraocular pressure and slit lamp fundoscopy); full blood count (FBC); urea and electrolytes (U\&Es); liver function tests (LFTs) and thyroid function tests (TFTs); serum pregnancy test for premenopausal women. Eligible patients will then be registered to the trial centrally via the University of Leeds automated $24 \mathrm{~h}$ system. Whenever possible, a biopsy of an identified iodine refractory lesion will be obtained following registration; archival tissue from initial thyroidectomy will also be requested.

\section{Treatment and assessment schedules}

Quality of life will be assessed within 28 days of registration for all participants, and tissue samples will be collected within the same timeframe where applicable. Participants will be asked to observe a low iodine diet from two weeks prior to receiving a baseline I-123 SPECT/CT scan which must be completed within 28 days of registration; $0.9 \mathrm{mg}$ recombinant human TSH (rhTSH) will be given via intramuscular administration two days and one day prior to administration of I-123. Prior to day one of treatment participants will have further assessments including repeat blood tests.

Participants will then be treated with $75 \mathrm{mg}$ of oral Selumetinib twice daily for four weeks; doses will be taken approximately $12 \mathrm{~h}$ apart. Repeat assessments including haematology and biochemistry will be conducted on day 14 and day 28; where applicable blood samples for cell-free DNA assessment will also be collected. An I-123 SPECT/CT scan at the end of treatment, day 28 ( \pm 1 day), will be reviewed centrally. The increase in uptake will be deemed significant if there is presence of any clinically relevant, targeted uptake in a lesion in which there was previously no uptake or there is an increase of 30\% or more in uptake in a lesion with evidence of some uptake on the baseline scan. Participants will be asked to recommence the low iodine diet two weeks prior to the post treatment scan and will again be administered $0.9 \mathrm{mg}$ rhTSH two days and one day prior to the scan. Participants will continue to observe the low iodine diet and take Selumetinib whilst the post treatment scan is undergoing 
Table 1 Eligibility criteria

Inclusion Criteria

- Diagnosed with locally advanced or metastatic differentiated thyroid cancer (papillary, follicular, Hürthle cell, or poorly differentiated carcinoma) with at least one measurable lesion as measured by computed tomography $(\mathrm{CT})$ or magnetic resonance imaging (MRI)

- Participants must have iodine refractory disease defined by one or more of the following:

- One or more measurable lesions that do not demonstrate iodine uptake on a previous radioiodine scan (diagnostic uptake or post therapy)

OR

- One or more measurable lesions that have progressed by RECIST 1.1 criteria within 12 months of I-131 therapy, despite demonstrable radioiodine avidity at the time of that treatment

- Participants must have radiological progression by RECIST 1.1 criteria within the prior 12 months

- Measurable disease by RECIST 1.1 criteria. Baseline scan must be completed within 4 weeks prior to the start of treatment.

- ECOG Performance Status $\leq 1$ and able to tolerate radioiodine therapy

- Life expectancy of at least 12 weeks

- Required laboratory values within 14 days of day 1 of treatment: - Adequate thyroid-stimulating hormone (TSH) suppression $<0.5 \mathrm{mU} / \mathrm{L}$

- Creatinine clearance $>50 \mathrm{ml} / \mathrm{min}$

- Absolute Neutrophil Count $\geq 1.5 \times 109 / L$ (1500 per mm3)

- Platelets $\geq 100 \times 109 / \mathrm{L}(100,000$ per mm3)

- Haemoglobin $>9.0 \mathrm{~g} / \mathrm{dL}$

- Serum bilirubin $\leq 1.5 \times$ upper limit of normal (ULN)

- Patients with no liver metastasis must have AST or

ALT $\leq 2.5 \times$ ULN

- Patients with liver metastasis must have AST or ALT $\leq 5 \times$ ULN.

If patients have AST or ALT $>3.5 \times$ ULN and $\leq 5 \times$ ULN they must have an $A L P \leq 6 \times$ ULN

- Able to give informed consent and willing to follow trial protocol.

- Aged over 18 years or over

- Female participants of child-bearing potential must have a negative pregnancy test within $24 \mathrm{~h}$ prior to starting therapy and agree to use dual methods of contraception for the duration of the trial and 6 months after completing treatment. Male participants must agree to use a barrier method of contraception for the duration of the trial and 4 months after completing treatment, if sexually active with a female of child-bearing potential.

- Able to swallow Selumetinib/l-131 capsules whole

Exclusion criteria

- Foci of anaplastic thyroid cancer

- Able to receive curative surgery or radiation therapy

- Major surgery (with the exception of surgical placement for vascular access), open biopsy, or significant traumatic injury $\leq 30$ days prior to registration

- Previous or concurrent cancer distinct in primary site or histology from thyroid cancer within previous 5 years, except for cervical cancer in situ, treated basal cell carcinoma, or squamous cell carcinoma of the skin or superficial bladder tumour

- Have received or are receiving an IMP or other systemic anticancer treatment within 4 weeks prior to the first dose of study treatment ( 6 weeks for nitrosoureas, mitomycin, and suramin), or within a period during which the IMP or anticancer treatment has not been cleared from the body (e.g. a period of 5 'half-lives'), whichever is the most appropriate and as judged by the investigator

- Any unresolved toxicity $\geq$ CTCAE Grade 2 from previous anti-cancer therapy, except for alopecia
Table 1 Eligibility criteria (Continued)

- Prior exposure to Tyrosine Kinase, MEK, RAS or RAF inhibitors

- Known or suspected allergy to Selumetinib or hypersensitivity to Selumetinib or any excipient agents or history of allergic reactions attributed to compounds of similar chemical or biologic composition to Selumetinib

- Have known or suspected brain metastases or spinal cord compression, unless the condition has been asymptomatic, has been treated with surgery and / or radiation, and has been stable without requiring corticosteroids nor anti-convulsant medications for at least 4 weeks prior to the first dose of study medication.

- Requiring medication with high iodine content (e.g. amiodarone)

- Participants who have had a lodine contrast enhanced CT scan in previous 2 months

- Ophthalmological conditions as follows:

- Intra-ocular pressure $>21 \mathrm{mmHg}$, or uncontrolled glaucoma (irrespective of intra-ocular pressure)

- Current or past history of retinal pigment epithelial detachment (REPD)/central serous retinopathy or retinal vein occlusion

- Any of the following cardiac conditions

- Uncontrolled hypertension (BP $\geq 150 / 95 \mathrm{mmHg}$ despite medical therapy)

- Acute coronary syndrome within 6 months prior to starting treatment

- Uncontrolled angina (Canadian Cardiovascular Society grade II-IV despite medical therapy)

- Symptomatic heart failure (NYHA grade II-IV), prior or current cardiomyopathy, or severe valvular heart disease

- Prior or current cardiomyopathy including but not limited to the following:

- Known hypertrophic cardiomyopathy

- Known arrhythmogenic right ventricular cardiomyopathy

- Severe valvular heart disease

- Left ventricular ejection fraction $<55 \%$ measured by echocardiography

- Atrial fibrillation with a ventricular rate $>100 \mathrm{bpm}$ on ECG at rest

- QTcF > $450 \mathrm{~ms}$ or other factors that increase the risk of QT prolongation

- Participants known to be infected with human immunodeficiency virus (HIV) or hepatitis B (HBV) or C (HCV) virus

- Any evidence of severe or uncontrolled systemic disease (e.g. unstable or uncompensated respiratory, cardiac, hepatic, or renal disease), active infection, active bleeding (including hepatitis B, hepatitis C, HIV), diatheses or renal transplant.

- Pregnant or breastfeeding females

- Male or female patients of reproductive potential and, as judged by the investigator, are not employing an effective method of birth control

- Have evidence of any other significant clinical disorder or laboratory finding that, as judged by the investigator, makes it undesirable for the patient to participate in the study.

- Hypersensitivity to bovine or human thyroid stimulating hormone or to any of the following excipients; mannitol, sodium phosphate monobasic, monohydrate, sodium phosphate dibasic, heptahydrate or sodium chloride.

- Hypersensitivity to I-123 or I-131 or to any of the following excipients; acetic acid, sodium hydroxide, sodium thiosulphate, sodium bicarbonate, sodium chloride, disodium phosphate anhydrous or silica

- Patients with dysphagia, oesophageal stricture, active gastritis, gastric erosions and peptic ulcer.

- Have refractory nausea and vomiting, chronic gastrointestinal diseases (e.g., inflammatory bowel disease), suspected reduced gastrointestinal motility or significant bowel resection that would adversely affect the absorption/bioavailability of orally administered study medication. 
review (for a maximum of 18 days). A further two to three SPECT/CT scans will also be acquired and used to predict the radiation dose that may be achieved with radioiodine treatment.

Participants not deemed to have sufficient increase in iodine uptake will cease treatment with Selumetinib, can return to a usual diet and will continue to be followed up for safety purposes until 30 days after completion of Selumetinib treatment. These individuals will receive no further trial treatment.

Participants with sufficient increase of iodine uptake will go on to receive treatment with I-131 and will continue to take Selumetinib until two days after administration of I-131 (if applicable) when the low iodine diet can be discontinued. rhTSH $0.9 \mathrm{mg}$ will be administered two days and one day prior to I-131 therapy. An activity of $5.5 \mathrm{GBq}( \pm 10 \%)$ I-131 sodium iodide will be administered orally. In the absence of any strong evidence of benefit from a dosimetric approach, it is felt reasonable to stick to empirical activities as per current standard UK practice. However, following I-131 therapy, dosimetry calculations will be made from three to four SPECT/CT scans. A standardised calibration protocol will be used by sites to optimise and standardise SPECT/ CT image data across participants. Therefore an essential element of the this trial is the need to set up, for the first time, a network of UK centres able to perform quantitative I-123 and high activity I-131 imaging.

For participants consenting to blood sample collection for PBI analysis, samples will be collected 24 and $144 \mathrm{~h}$ post I-131 therapy.

Upon completion of I-131 therapy, participants will be followed up quarterly for the first 12 months and then 6 monthly for the duration of the trial (i.e. until the last patient recruited reaches 12 months follow up) or until evidence of disease progression is confirmed by CT scan. Follow-up assessments will include measurement of thyroglobulin and antithyroglobulin antibodies; thyroid function test; CT neck, thorax, abdomen and pelvis. Quality of life assessments will be repeated at each follow up for the first 12 months, and where applicable blood sample collection for cell-free DNA assessment will occur at three months and six months.

For all participants, TSH suppression to less than 0.1 units should be maintained throughout treatment and follow-up. Also, for all participants (including those not receiving I-131 therapy), assessments at 30 days post end of Selumetinib treatment will include vital signs, 12-lead ECG, haematology, echocardiogram and repeat quality of life measures.

The participant pathway can be seen schematically in Fig. 1, and Table 2 (SPIRIT diagram) details the assessment schedule.

\section{Outcomes}

\section{Primary endpoint}

The primary endpoint of the trial is PFS at 12 months, reported as the percentage of participants alive and progression-free at 12 months. PFS is calculated from date of registration to first documented evidence of disease progression or death. Participants who have not progressed at the time of analysis will be censored at the last date alive and progression-free.

\section{Secondary endpoints}

- Safety: Serious adverse events (SAEs) from registration until 30 days post cessation of trial therapy (Selumetinib or I-131 therapy); suspected unexpected serious adverse reactions (SUSARs) and serious adverse reaction (SARs) recorded for all from start of protocol treatment for the lifetime of the trial;

- Toxicity: Adverse reactions (ARs) as graded by common terminology criteria for adverse events (CTCAE) v4.0;

- Overall survival (OS): calculated from date of registration to date of death; censoring will occur at last known date alive for those alive at time of analysis;

- Sufficient iodine uptake: reviewed centrally; sufficient uptake is defined as presence of any clinically relevant uptake in a lesion in which there was previously no uptake or an increase of $30 \%$ or more in uptake in a lesion with evidence of some uptake on the baseline scan;

- Radiological response: assessed via CT scans and based on response evaluation criteria in solid tumours (RECIST) v1.1.

\section{Exploratory endpoints}

- Lesional and whole body dosimetry: As measured from a minimum of three and maximum of four SPECT/CT scans over six days following Selumetinib and I-131;

- BRAF, NRAS and TERT gene mutational status and additional relevant genes: from circulating cell-free DNA, iodine refractory tissue samples and historic tissue samples where available;

- PBI levels from blood samples;

- Quality of life: assessed using QLQ-C30 plus head and neck cancer supplementary questions (H\&N35), and EQ-5D ${ }^{\mathrm{m}}$;

- Change in serum thyroglobulin levels: assessed via percentage change from baseline at each time point.

\section{Analysis}

Statistical analysis for final and interim analyses are fully detailed in separate analysis plans. The full analysis set will include all registered participants receiving at least one 


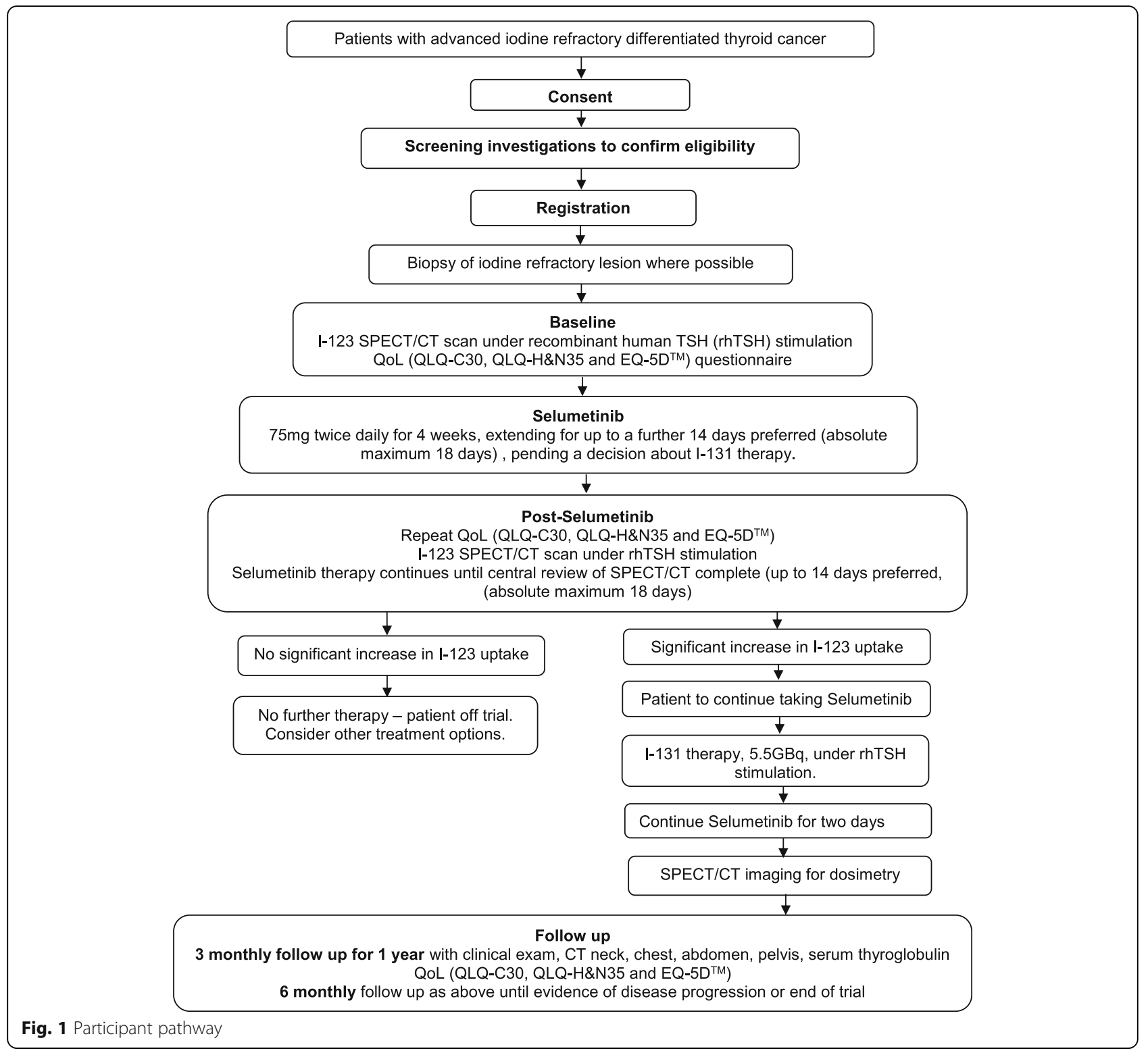

dose of Selumetinib and will be equivalent to the safety analysis set. The iodine uptake (IU) cohort will include participants from the full analysis set who were deemed to have sufficient I-123 uptake following treatment with Selumetinib. All other participants in the full analysis set will be the non-IU cohort. All efficacy endpoint analyses will be conducted on the IU cohort only, unless otherwise specified in the analysis plan. Safety analyses will be conducted on the safety analysis set with data summarised for each study cohort and overall during initial therapy.

\section{Primary endpoint analysis}

The proportion of participants alive and progression free at 12 months will be presented with corresponding confidence intervals (CI). The primary analysis will test the hypothesis that PFS in the IU cohort is less than or equal to $25 \%$, against an alternative of $44 \%$ based on the Nelson-Aalen estimate of the cumulative hazard function at 12 months, following the methodology of Case and Morgan [27].

\section{Secondary endpoint analyses}

SAEs, SARs and SUSARs will be reported overall and by treatment period (Selumetinib, I-131) for all participants and for the IU and non-IU cohorts.

The number and proportion of participants experiencing each grade of toxicity will be summarised in the same analysis sets. 
Table 2 Minimum schedule of assessments (SPIRIT diagram)

\begin{tabular}{|c|c|c|c|c|c|c|c|c|}
\hline & \multicolumn{2}{|l|}{ Baseline } & \multicolumn{4}{|c|}{ Treatment } & \multirow{3}{*}{$\begin{array}{l}\text { End of Treatment } \\
30 \text { days post end } \\
\text { of Selumetinib } \\
\text { treatment }\end{array}$} & \multirow{3}{*}{$\begin{array}{l}\text { Follow Up } \\
3 \text { monthly for first } \\
12 \text { months then } 6 \\
\text { monthly for duration } \\
\text { of trial }^{b}\end{array}$} \\
\hline & \multirow{2}{*}{$\begin{array}{l}\text { Within } 14 \text { days } \\
\text { prior to registration }\end{array}$} & \multirow{2}{*}{$\begin{array}{l}\text { Within } 28 \text { days } \\
\text { of registration }\end{array}$} & \multicolumn{3}{|c|}{ Selumetinib } & \multirow{2}{*}{$\begin{array}{l}\text { I-131 } \\
\text { Therapy }\end{array}$} & & \\
\hline & & & Day 1 & Day 14 & Day 28 & & & \\
\hline Informed consent & $x$ & & & & & & & \\
\hline $\begin{array}{l}\text { Patient details (initials, date } \\
\text { of birth, sex) }\end{array}$ & $x$ & & & & & & & \\
\hline Medical History & $x$ & & & & & & & \\
\hline Physical Examination/Vital signs & $x$ & & $x$ & $x$ & $x$ & & $x$ & $x$ \\
\hline Ophthalmologic assessment & $x$ & & & & & & & \\
\hline 12-Lead ECG & $x$ & & $x$ & & & & $x$ & \\
\hline Laboratory tests & $x$ & & $x$ & $x$ & $x$ & & $x$ & $x$ \\
\hline Pregnancy test & $x$ & & $x$ & & & $x$ & $x$ & $X^{c}$ \\
\hline Echocardiography /MUGA & $x$ & & $x$ & & & & & \\
\hline Dispensing of Selumetinib & & & $x$ & & & & & \\
\hline Tissue collection & & $x$ & & & & & & \\
\hline $\begin{array}{l}\mathrm{CT} \text { neck, thorax, abdomen } \\
\text { and pelvis }\end{array}$ & $x^{a}$ & & & & & & & $x$ \\
\hline I-123 SPECT/CT & & $x$ & & & $x$ & & & \\
\hline $\begin{array}{l}\text { SPECT/CT for Lesion Dosimetry } \\
\text { post I-131 therapy }\end{array}$ & & & & & & $x$ & & \\
\hline Adverse Events & $x$ & & $x$ & $x$ & $x$ & $x$ & $x$ & $x^{d}$ \\
\hline Concomitant Medications & $x$ & & $x$ & $x$ & $x$ & & $x$ & \\
\hline $\begin{array}{l}\text { Quality of life (QLQ-C30, } \\
\text { QLQ-H\&N35 and EQ-5D'M) }\end{array}$ & & $x$ & & & & & $x$ & $x^{e}$ \\
\hline $\begin{array}{l}\text { Protein bound iodine blood } \\
\text { sample collection }\end{array}$ & & & & & & $x^{f}$ & & \\
\hline $\begin{array}{l}\text { Cell-free DNA blood sample } \\
\text { collection }\end{array}$ & & & $x$ & $x$ & $x$ & & $x$ & $X^{9}$ \\
\hline
\end{tabular}

${ }_{\mathrm{a}}$ CT scans have not been performed within 28 days of starting treatment, an additional CT neck, chest, abdomen, and pelvis must be performed to enable assessment of response and progression-free survival as per RECIST criteria

${ }^{b}$ Participants who do not proceed to radioiodine therapy will be followed up for safety purposes until 30 days after completion of Selumetinib treatment only

'Pregnancy test to be done at the first and second 3 monthly follow up visits ( 3 month and 6 month follow-up)

${ }^{\mathrm{d}}$ Adverse reactions only at follow up visits

e Quality of life is assessed at baseline, end of treatment and 3 monthly for the first 12 months of follow-up

${ }^{\mathrm{f}}$ Collected 24 and $144 \mathrm{~h}$ following $\mathrm{l}-131$ dosing

$g_{3}$ and 6 months only

OS will be summarised using Kaplan-Meier curves; OS at 12 months and median OS will both be presented alongside CIs.

The proportion of participants with sufficient iodine uptake to progress to I-131 will be presented with corresponding 95\% CI. This will also be presented excluding individuals who did not receive Selumetinib for seven days prior to the scan.

The proportion of participants in the IU cohort achieving at least a partial response will be presented with 95\% CIs. The proportion of participants in each best response category (complete response, partial response, stable disease, and progressive disease) will also be presented.

\section{Exploratory endpoint analyses}

The absorbed dose to each lesion, whole body and blood measurements will be presented descriptively alongside measures of uncertainty. Regression modelling will be used to investigate association between these doses and outcome as appropriate. Analogous analyses will explore association between lesional absorbed dose, radiological response and overall survival, as will analyses considering whole body and blood absorbed doses and grades of toxicity.

Gene mutational status will be summarised for all available samples. PFS and response will be summarised by gene mutational status. 
PBI measurements will be summarised for all participants receiving I-131 with samples available. The relationship between absorbed dose delivered and treatment outcome will be investigated.

Quality of life will be summarised with 95\% CIs adjusting for baseline mean scores. The QLQ-C30 and H\&N35 will be summarised for each domain. The proportion of participants scoring each category for the five EQ-5D ${ }^{\text {ts }}$ domains will be presented.

Mean change in thyroglobulin from baseline at each time point will be presented with corresponding standard error. Mean change will also be presented by maximum RECIST response. A waterfall plot of maximum change per participant will be presented.

\section{Trial and data monitoring}

A Trial Steering Committee will be convened to periodically review safety data; an independent Data Monitoring and Ethics Committee (DMEC) will review safety data to determine patterns and trends of events which would not be apparent on an individual case basis. Following the interim analysis, the DMEC may request that the trial be terminated for lack of activity if there is evidence to suggest that 12 month PFS is no better than $25 \%$ based on the Nelson-Aalen estimator.

\section{Discussion}

The SEL-I-METRY trial will investigate a potential treatment to resensitise radioiodine refractory patients with differentiated thyroid cancer. Current treatment options for this group of patients, the multi-targeted kinase inhibitors Sorafenib and Lenvatinib, need to be given continuously and are associated with significant toxicity and hence may be detrimental to quality of life.

Patients who have radioiodine avid disease have better survival outcomes and thus resensitising refractory patients could lead to improvements in progression-free survival without the need for continuous treatment and the associated toxicity. There are already considerable data regarding the tolerability of the Selumetinib regimen, and a pilot trial found an indication of an increase in iodine uptake. Both toxicity and efficacy will be assessed in this larger Phase II multicentre trial.

If the SEL-I-METRY trial demonstrates that Selumetinib followed by further I-131 NaI is a useful treatment strategy for this group of patients, a randomised trial comparing this approach with other treatments, such as the multi-kinase inhibitors, could be considered to help define the optimal treatment for these patients.

In addition, an essential element of this trial is the need to set up, for the first time, a network of UK centres able to perform quantitative high activity I-131 imaging which is essential for dosimetric calculations. This information will be collected to investigate prediction of response to
I-131, presenting an unprecedented opportunity to investigate the role of lesional dosimetry in molecular radiotherapy, leading to greater personalisation of therapy, both with I-131 and with other isotope therapies.

\section{Trial status}

At the time of submission, SEL-I-METRY is open to recruitment.

\section{Additional file}

Additional file 1: Patient Imaging and Dosimetry Protocol. (DOC 19 kb)

Additional file 2: Recommended items to address in a clinical trial protocol and related documents*. (DOC $122 \mathrm{~kb}$ )

\section{Abbreviations}

AE: Adverse event; AR: Adverse reaction; Cl: Confidence intervals; CTCAE: Common terminology criteria for adverse events;

EGC: Electrocardiogram; FBC: Full blood count; GBq: Giga Becquerel; Gy: Gray; I-123: lodine-123; I-124: lodine-124; I-131: lodine-131; IU: lodine uptake; LFT: Liver function test; MAPKinase: Mitogen-activated protein kinase; MUGA: Multiple gated acquisition; Nal: Sodium iodide; NIS: Sodium iodide symporter; OS: Overall survival; PBI: Protein bound iodine; PET: Positron emission tomography; PFS: Progression free survival; RAl: Radioactive iodine; rhTSH: Recombinant human thyroid stimulating hormone; SAE: Serious adverse event; SPECT/CT: Single photon emission computed tomography/ computed tomography; SPIRIT: Standard protocol items: recommendations for interventional trials; SUSAR: Suspected unexpected serious adverse reaction; TFT: Thyroid function tests; TSH: Thyroid stimulating hormone; U\&Es: Urea and electrolytes; UK: United Kingdom

\section{Funding}

The trial is sponsored by Sheffield Teaching Hospitals NHS Foundation Trust. The study is funded by Cancer Research UK and supported by Astra Zeneca. The funding bodies reviewed the design of the study, the sponsor had no involvement in design. Neither the funding bodies nor the sponsor will have a role in the collection, analysis or interpretation of data and neither had an input into the writing of this manuscript.

The Investigational Medicinal Product in this trial (Selumetinib) is provided by AstraZeneca free of charge for this study. rhTSH is being provided by Genzyme free of charge. Discounted I-123 is being provided by GE Healthcare. RG is funded by the National Institute for Health Research (NIHR) Imaging Clinical Research Facility at the Royal Marsden NHS Foundation Trust.

Availability of data and materials Not applicable.

\section{Authors' contributions}

The study was designed by JW, LM, KN, GF, YD, KF, DGdC, SB and LF. AH and $\mathrm{SB}$ developed the statistical analysis plan. $\mathrm{HB}, \mathrm{SB}$, JW wrote the manuscript based on version 5.0 of the protocol (21/12/17). JW, LM, KN, GF, YD, RG and DGdC perform the research and collect data. LF performs trial and data management. $\mathrm{AH}, \mathrm{HB}$ and $\mathrm{SB}$ are statisticians. All authors reviewed and approved the final manuscript.

Ethics approval and consent to participate

The trial has received ethics approval from the NHS National Research Ethics Service (NRES) East Midlands-Leicester South (15/EM/0455). All participants provide full informed consent to participate in SEL-I-METRY.

\section{Consent for publication}

Not applicable.

\section{Competing interests}

$\mathrm{SRB}, \mathrm{AH}, \mathrm{HB}, \mathrm{LF}, \mathrm{KF}, \mathrm{LM}, \mathrm{RG}, \mathrm{YD}$ and $\mathrm{GF}$ declare that they have no competing interests. JW has previously received honoraria for attending Advisory Boards for both AstraZeneca and Sanofi-Genzyme and has received honoraria/support 
to attend educational meetings from Eisai, Bayer and Sobi. KN has received honoraria for teaching and consultancy from Eisai, AstraZeneca, SanofiGenzyme and Sobi. DGdC has received honoraria, consultancy and speaker fees as well as research funding from AstraZeneca.

\section{Publisher's Note}

Springer Nature remains neutral with regard to jurisdictional claims in published maps and institutional affiliations.

\section{Author details}

'Leeds Institute of Clinical Trial Research, University of Leeds, Leeds LS2 9JT, UK. ${ }^{2}$ Centre for Cancer Research and Cell Biology, Queen's University Belfast, Belfast BT9 7BL, Northern Ireland, UK. ${ }^{3}$ Butterfly Thyroid Cancer Trust, NCCC Freeman Hospital, Newcastle NE39 2PU, UK. ${ }^{4}$ Velindre Cancer Centre, Cardiff CF14 2TL, UK. ${ }^{5}$ Joint Department of Physics, The Royal Marsden NHS Foundation Trust and Institute of Cancer Research, Sutton SM2 5PT, UK. ${ }^{6}$ The Royal Marsden NHS Foundation Trust, Sutton SM2 5PT, UK. 'Department of Nuclear Medicine, The Royal Marsden NHS Foundation Trust, Sutton SM2 5PT, UK. ${ }^{8}$ Weston Park Hospital, Sheffield S10 2SJ, UK.

Received: 21 March 2019 Accepted: 28 March 2019

Published online: 14 June 2019

\section{References}

1. CRUK. Thyroid cancer statistics [cited 2017 July]. Available from: http://www. cancerresearchuk.org/health-professional/cancer-statistics/statistics-bycancer-type/thyroid-cancer. Accessed 7 May 2019.

2. Durante C, Haddy N, Baudin E, Leboulleux S, Hartl D, Travagli J, et al. Longterm outcome of 444 patients with distant metastases from papillary and follicular thyroid carcinoma: benefits and limits of radioiodine therapy. The Journal of Clinical Endocrinology \& Metabolism. 2006;91(8):2892-9.

3. Liu Y, Van Der Pluijm G, Karperien M, Stokkel M, Pereira A, Morreau J, et al. Lithium as adjuvant to radioiodine therapy in differentiated thyroid carcinoma: clinical and in vitro studies. Clin Endocrinol. 2006;64(6):617-24.

4. Coelho SM, Corbo R, Buescu A, Carvalho D, Vaisman M. Retinoic acid in patients with radioiodine non-responsive thyroid carcinoma. J Endocrinol Investig. 2004;27(4):334-9.

5. Brose MS, Nutting CM, Jarzab B, Elisei R, Siena S, Bastholt L, et al. Sorafenib in radioactive iodine-refractory, locally advanced or metastatic differentiated thyroid cancer: a randomised, double-blind, phase 3 trial. Lancet. 2014; 384(9940):319-28.

6. Schlumberger M, Tahara M, Wirth $L$, Robinson B, Brose MS, Elisei R, et al. Lenvatinib versus placebo in radioiodine-refractory thyroid Cancer. New Engl J Med. 2015;372(7):621-30.

7. Cohen J, Xing MZ, Mambo E, Guo ZM, Wu GG, Trink B, et al. BRAF mutation in papillary thyroid carcinoma. J Natl Cancer I. 2003;95(8):625-7.

8. Durante C, Puxeddu E, Ferretti E, Morisi R, Moretti S, Bruno R, et al. BRAF mutations in papillary thyroid carcinomas inhibit genes involved in iodine metabolism. J Clin Endocr Metab. 2007;92(7):2840-3.

9. Liu DX, Hu SY, Hou P, Jiang D, Condouris S, Xing MZ. Suppression of BRAF/ MEK/MAP kinase pathway restores expression of iodide-metabolizing genes in thyroid cells expressing the V600E BRAF mutant. Clin Cancer Res. 2007; 13(4):1341-9.

10. Bodoky G, Timcheva C, Spigel DR, La Stella PJ, Ciuleanu TE, Pover G, et al. A phase II open-label randomized study to assess the efficacy and safety of selumetinib (AZD6244 [ARRY-142886]) versus capecitabine in patients with advanced or metastatic pancreatic cancer who have failed first-line gemcitabine therapy. Investig New Drugs. 2012;30(3):1216-23.

11. Carvajal RD, Schwartz GK, Mann H, Smith I, Nathan PD. Study design and rationale for a randomised, placebo-controlled, double-blind study to assess the efficacy of selumetinib (AZD6244; ARRY-142886) in combination with dacarbazine in patients with metastatic uveal melanoma (SUMIT). BMC Cancer. 2015;15(1):1

12. Carvajal RD, Sosman JA, Quevedo JF, Milhem MM, Joshua AM, Kudchadkar $\mathrm{RR}$, et al. Effect of selumetinib vs chemotherapy on progression-free survival in uveal melanoma: a randomized clinical trial. Jama. 2014;311(23):2397-405.

13. Catalanotti F, Solit DB, Pulitzer MP, Berger MF, Scott SN, lyriboz T, et al. Phase II trial of MEK inhibitor selumetinib (AZD6244, ARRY-142886) in patients with BRAFV600E/K-mutated melanoma. Clin Cancer Res. 2013;19(8):2257-64.
14. Gupta A, Love S, Schuh A, Shanyinde M, Larkin J, Plummer R, et al. DOC-MEK: a double-blind randomized phase II trial of docetaxel with or without selumetinib in wild-type BRAF advanced melanoma. Ann Oncol. 2014;25(5):968-74.

15. Kirkwood JM, Bastholt L, Robert C, Sosman J, Larkin J, Hersey P, et al. Phase II, open-label, randomized trial of the MEK1/2 inhibitor selumetinib as monotherapy versus temozolomide in patients with advanced melanoma. Clin Cancer Res. 2012;18(2):555-67.

16. Robert C, Dummer R, Gutzmer R, Lorigan P, Kim KB, Nyakas M, et al. Selumetinib plus dacarbazine versus placebo plus dacarbazine as first-line treatment for BRAF-mutant metastatic melanoma: a phase 2 double-blind randomised study. The lancet oncology. 2013;14(8):733-40.

17. Jänne P, Smith I, McWalter G, Mann H, Dougherty B, Walker J, et al. Impact of KRAS codon subtypes from a randomised phase II trial of selumetinib plus docetaxel in KRAS mutant advanced non-small-cell lung cancer. $\mathrm{Br} J$ Cancer. 2015;113(2):199-203.

18. Jänne PA, Mann H, Ghiorghiu D. Study design and rationale for a randomized, placebo-controlled, double-blind study to assess the efficacy and safety of Selumetinib in combination with docetaxel as second-line treatment in patients with KRAS-mutant advanced non-small cell lung Cancer (SELECT-1). Clinical lung cancer. 2016;17(2):e1-4.

19. Jänne PA, Shaw AT, Pereira JR, Jeannin G, Vansteenkiste J, Barrios C, et al. Selumetinib plus docetaxel for KRAS-mutant advanced non-small-cell lung cancer: a randomised, multicentre, placebo-controlled, phase 2 study. The lancet oncology. 2013;14(1):38-47.

20. Hayes DN, Lucas AS, Tanvetyanon T, Krzyzanowska MK, Chung CH, Murphy BA, et al. Phase II efficacy and pharmacogenomic study of Selumetinib (AZD6244; ARRY-142886) in iodine-131 refractory papillary thyroid carcinoma with or without follicular elements. Clin Cancer Res. 2012;18(7): 2056-65.

21. Ho AL, Grewal RK, Leboeuf R, Sherman EJ, Pfister DG, Deandreis D, et al. Selumetinib-enhanced radioiodine uptake in advanced thyroid cancer. N Engl J Med. 2013;368(7):623-32.

22. Wilky BA, Rudek M, Ahmed S, Laheru D, Cosgrove D, Donehower RC, et al. A phase I trial of vertical inhibition of IGF signalling using cixutumumab, an anti-IGF-1R antibody, and selumetinib, an MEK 1/2 inhibitor, in advanced solid tumours. Br J Cancer. 2015;112(1):24-31.

23. Perros P, Boelaert K, Colley S, Evans C, Evans RM, Gerrard G, et al. Special issue: British thyroid association guidelines for the Management of Thyroid Cancer Introduction. Clin Endocrinol. 2014:81:1-122.

24. McGowan DR, Guy MJ. Time to demand dosimetry for molecular radiotherapy? Brit J Radiol. 2015;88(1047).

25. Flux GD, Haq M, Chittenden SJ, Buckley S, Hindorf C, Newbold K, et al. A dose-effect correlation for radioiodine ablation in differentiated thyroid cancer. Eur J Nucl Med Mol Imaging. 2010;37(2):270-5.

26. Chan A, Tetzlaff JM, Altman DG, Laupacis A, Gøtzsche PC, Krleža-Jerić K, et al. SPIRIT 2013 Statement: Defining Standard Protocol Items for Clinical Trials. Ann Intern Med. 2013;158:200-207.

27. Case L, Morgan TM. Design of phase II cancer trials evaluating survival probabilities. BMC Med Res Methodol. 2003;3(1):1.

Ready to submit your research? Choose BMC and benefit from:

- fast, convenient online submission

- thorough peer review by experienced researchers in your field

- rapid publication on acceptance

- support for research data, including large and complex data types

- gold Open Access which fosters wider collaboration and increased citations

- maximum visibility for your research: over $100 \mathrm{M}$ website views per year

At BMC, research is always in progress.

Learn more biomedcentral.com/submissions 